Published in "Colloids and Surfaces A:

Physicochemical and Engineering Aspects 445: 21-29, 2014"

which should be cited to refer to this work.

\title{
A closer look at the synthesis and formation mechanism of hematite nanocubes
}

\author{
Vikash Malik $^{\mathrm{a}}$, Bernard Grobety ${ }^{\mathrm{b}}$, Veronique Trappe ${ }^{\mathrm{c}}$, Herve Dietsch $^{\mathrm{a}}$, \\ Peter Schurtenberger ${ }^{\mathrm{d}, *}$ \\ a Adolphe Merkle Institute (AMI) and Fribourg Center for Nanomaterials (FriMat), University of Fribourg, CH-1723 Marly, Switzerland \\ b Department of Geosciences, University of Fribourg, CH-1700 Fribourg, Switzerland \\ ' Department of Physics, University of Fribourg, CH-1700 Fribourg, Switzerland \\ d Physical Chemistry, Department of Chemistry, Lund University, SE-22100, Sweden
}

\section{H I G H L I G H T S}

- We synthesized hematite cubeshaped nanoparticles using different synthesis paths.

- Iron chloride concentration and akaganeite concentration influence morphology and particle size.

- We use electron microscopy and diffraction methods to characterize the particles.

- Controlling the akaganeite concentration and size is key to tune particle size and crystallinity.

- We discuss possible formation mechanisms of single and polycrystalline hematite nanocubes.
G R A P H I C A L A B S T R A C T

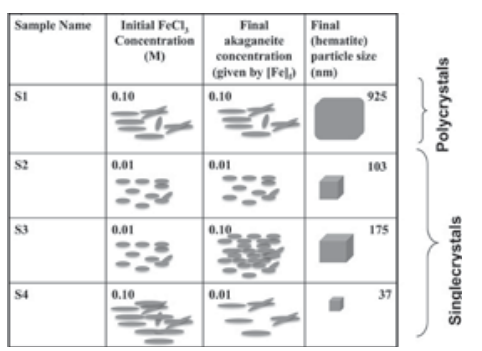

We have synthesized hematite cube-shaped nanoparticles using different synthesis paths to examine the effect of the intermediate product akaganeite $(\beta-\mathrm{FeOOH})$ on the size and shape of the final hematite particles. Akaganeite spindles and hematite nanocubes are prepared using forced hydrolysis of iron chloride $\left(\mathrm{FeCl}_{3}\right)$ salt. We use a combination of transmission electron microscopy (TEM), high resolution transmission electron microscopy (HR-TEM), selected area electron diffraction(SAED) and dynamic light scattering (DLS). Akaganeite particles are found to be spindles, with an average length that depends linearly on the $\mathrm{FeCl}_{3}$ concentration, while their aspect ratio is hardly affected by the same parameter. Adjusting the akaganeite concentration from $0.01 \mathrm{M}$ to $0.10 \mathrm{M}$ equivalent of $\mathrm{FeCl}_{3}$ in the subsequent transformation to hematite leads to single crystal nanocubes with sizes ranging from 37 to $175 \mathrm{~nm}$. The combination of akaganeite concentration and size is found to be the key parameter to control the size and crystallinity of the resulting hematite particles. Possible formation mechanisms of single and polycrystalline hematite nanocubes are discussed.

\footnotetext{
* Corresponding author. Tel.: +46 46 2228219; fax: +46 462224413.

E-mail address: peter.schurtenberger@fkem1.lu.se (P. Schurtenberger).
}

\section{Introduction}

Colloidal nanoparticles are increasingly important for applications in biomedicine [1], imaging [2], DNA separation [3], colloidal crystallization [4-6] and numerous other areas. Controlled synthesis techniques are required to obtain monodisperse colloids 
with tunable size, shape, physical and chemical properties. Particularly well-known and increasingly studied classes of particles are oxides and hydroxides of iron, for example hematite, magnetite, maghemite, and goethite. Hematite $\left(\alpha-\mathrm{Fe}_{2} \mathrm{O}_{3}\right)$ shows very interesting magnetic properties, which change with particle size and shape. Nanosized hematite has been synthesized in various shapes such as cubes [7-9], rods [10], spheres [11,12], pseudocubes [12], platelets [13], ellipsoids [14,15], nanobelts and dendrite micro pines [16,17]. The final hematite particle size and shape can be tuned by adjusting synthesis parameters such as the concentration of the reactants [7], duration of the reaction [18], ionic strength [19,20], reaction temperature, surfactants [10], presence of hematite seed [21] and $\mathrm{pH}$ of the solution [19]. The addition of organic additives to aqueous reaction mixtures was shown to have an appreciable effect on the geometry of hematite particles [22]. Changing the ratio of solvent mixtures strongly influences the final shape, yielding spindle, rhombohedral, or cubical geometries particularly in the case of ethylene diamine/ethanol mixtures [9].

Fine-tuning can be achieved by the addition of sulphate and phosphate salts to the reaction mixture. These salts restrict the growth of hematite nuclei in the direction perpendicular to the $c$-axis $[19,20]$. The adsorption of sulphate ions has been found to depend on the $\mathrm{pH}$ of the solution, and was reported to decrease with increasing $\mathrm{pH}$ [19].When dissolved in water at $100^{\circ} \mathrm{C}, \mathrm{FeCl}_{3}$ initially forms spindle shaped akaganeite $(\beta-\mathrm{FeOOH})$ particles [23]. Hematite particles are then grown via the dissolution and reprecipitation of this intermediate metastable phase. In order to get full control over the formation of hematite, it is essential to understand the formation and interparticle interactions of akaganeite. In this context it is also important to realize that akaganeite spindles form diverse aggregates such as stacks depending on the balance between repulsive and attractive forces between them [24], which can influence the resulting size, shape and structure of hematite particles formed.

The formation mechanisms of differently shaped hematite particles from akaganeite precursors were studied in the past by Bailey and Mercartney as a function of the $\mathrm{FeCl}_{3}$ concentration $(0.018-0.45 \mathrm{M})$ [12]. They described in particular the formation of polycrystalline pseudocubical hematite particles (i.e. cubes with round edges) at high $\mathrm{FeCl}_{3}$ concentration through a three-step process: first, akaganeite rods are formed, which then arrange into stacks, finally leading to pseudocubes [12]. An important observation made was that these particles were found to be polycrystalline. The polycrystalline nature of the pseudocubes was related to the fact that nucleation of hematite occurs at the akaganeite interface, and a model for the formation mechanism was developed based on Debye-Huckel theory [25].Given the importance of single crystal nanoparticles for various applications and the fact that the characterization of typical materials properties is generally easier for single crystal particles [26-29], we thus started an investigation of the formation hematite particles at low $\mathrm{FeCl}_{3}$ concentrations (0.01-0.1 M). Moreover, in our attempt to achieve full control over the nanostructure of hematite particles we extended this study also to an investigation of the influence of the size and concentration of the metastable akaganeite precursor particles on the crystallinity and size of the final hematite particles.

\section{Materials and methods}

Reagent grade $\mathrm{FeCl}_{3}$ was purchased from Merck and used without further purification. MilliQ water with a resistivity of $18.2 \mathrm{M} \Omega \mathrm{cm}$ was used for all the syntheses. Carbon coated grids (300 mesh) from Electron Microscopy Services Inc. were used for the preparation of the TEM grids and the investigations of the particle morphology.

\subsection{Characterization techniques}

X-ray powder diffraction (XRD) patterns of the products were measured on a X-ray diffractometer (XRD-IC100P) in the $2 \theta$ range of $10-80^{\circ}$. A transmission electron microscope (TEM-CM200, Philips) operating at $200 \mathrm{keV}$ was used for size and morphology studies, selected area electron diffraction (SAED) analysis and high resolution TEM (HRTEM) imaging of the individual particles. A typical sample for TEM was prepared by concentrating the particle dispersion using a centrifuge at $5000 \mathrm{rpm}$ and drying one droplet of $1 \mathrm{vol} \%$ of particles onto a carbon coated ( 300 mesh) grid. For the size distribution of nanoparticles in a liquid suspension, dynamic light scattering (3DDLS goniometer system from LS Instruments Inc.) was employed. Intensity cross-correlation functions were analyzed using a second order cumulant expansion. Nanoparticle suspensions ( $0.01 \mathrm{vol} \%$ in water) were measured in a cylindrical DLS cuvette $(10 \mathrm{~mm}$ diameter) at room temperature. The scattering angle was kept at $90^{\circ}$. We were not able to accurately determine the hydrodynamic radius of $\mathrm{S} 1$ due to the fast sedimentation of the large particles in the DLS tube.

\subsection{Synthesis}

For the preparation of akaganeite, solutions of $\mathrm{FeCl}_{3}$ with concentrations of $0.01,0.02,0.04,0.08$ and $0.10 \mathrm{M}$ were prepared by dissolving the appropriate amount of $\mathrm{FeCl}_{3}$ (162 mg-1.62 g) into $100 \mathrm{~mL}$ of MilliQ water. These reaction mixtures were kept in a preheated oven at $98^{\circ} \mathrm{C}$ for $1 \mathrm{~h}$ and then quenched to room temperature by inserting the samples into a water bath at room temperature.

We followed the synthesis of hematite in (1) a one step process, starting from $\mathrm{FeCl}_{3}$ solutions at two initial concentrations (S1: $0.1 \mathrm{M}$; and S2: $0.01 \mathrm{M}$ ), and (2) in a two-step process, where the reaction was quenched after the production of the metastable akaganeite precursor.

One-step process: For sample $\mathrm{S} 1\left(\mathrm{FeCl}_{3}\right.$ concentration $\left.0.10 \mathrm{M}\right)$, $1.62 \mathrm{~g} \mathrm{FeCl}_{3}$ was dissolved in $100 \mathrm{~mL}$ water, for sample $\mathrm{S} 2\left(\mathrm{FeCl}_{3}\right.$ concentration $0.01 \mathrm{M}$ ), $356 \mathrm{mg}$ of $\mathrm{FeCl}_{3}$ was dissolved into $220 \mathrm{~mL}$ of water. Sample S1 was divided into four glass bottles of equal volume, which were kept at the reaction temperature for $1 \mathrm{~h}, 4 \mathrm{~h}$, $24 \mathrm{~h}$ and $192 \mathrm{~h}$, respectively. Sample S2 was divided into eleven tightly closed glass bottles of equal volume, which were removed from the oven after reaction times ranging from $5 \mathrm{~min}$ up to $192 \mathrm{~h}$. All samples were quenched into a water bath at room temperature after these different reaction durations. The products were then concentrated and characterized by TEM.

Two-step process: In the two-step process, two stock solutions of respectively $0.01 \mathrm{M}$ (sample S3) and $0.10 \mathrm{M}$ (sample S4) of $\mathrm{FeCl}_{3}$ in water were prepared. The concentration of akaganeite particles was then adjusted and the reaction was started again (S3: initial $\mathrm{FeCl}_{3}$ concentration $0.01 \mathrm{M}$, final concentration $0.1 \mathrm{M}$; and S4: initial $\mathrm{FeCl}_{3}$ concentration $0.1 \mathrm{M}$, final concentration $0.01 \mathrm{M}$ ).

One liter of $\mathrm{S} 3$ solution was kept in the preheated oven at $98^{\circ} \mathrm{C}$ for $1 \mathrm{~h}$ and quenched to room temperature to stop the reaction, then $90 \%(900 \mathrm{~mL})$ of the water was removed from the quenched akaganeite solution using a rotary evaporator at $50^{\circ} \mathrm{C}$ under reduced pressure. The resulting solution was considered to have an equivalent iron chloride concentration of $0.10 \mathrm{M}$. This solution was labelled as $0 \mathrm{~h}$ solution. It was divided into five batches with equal volume $(20 \mathrm{~mL})$, kept in the preheated oven at $98^{\circ} \mathrm{C}$ and removed after different periods of time (1-192 h).

In parallel, five batches of $5 \mathrm{~mL}$ of S4 solution were kept in the preheated oven for $1 \mathrm{~h}$ (labelled as $0 \mathrm{~h}$ solution). To each batch $45 \mathrm{~mL}$ of preheated $\left(98^{\circ} \mathrm{C}\right)$ deionized water was added after $1 \mathrm{~h}$ of reaction, diluting all batches of the $\mathrm{S} 4$ starting solution by a factor of 10 , which leads to a solution with an equivalent concentration of 
Table 1

Description of initial $\left(\left[\mathrm{FeCl}_{3}\right]_{\text {ini }}\right)$ and final $\left(\left[\mathrm{FeCl}_{3}\right]_{f}\right)$ concentration of $\mathrm{FeCl}_{3}$. TEM derived and hydrodynamic (DLS) particle size are also shown for all experiments.

\begin{tabular}{llllc}
\hline Sample & {$\left[\mathrm{FeCl}_{3}\right](\mathrm{M})$} & {$\left[\mathrm{FeCl}_{3}\right]_{f}(\mathrm{M})$} & $\begin{array}{l}d(\mathrm{~nm}) \text { from } \\
\text { TEM }\end{array}$ & $\begin{array}{c}R_{h}(\mathrm{~nm}) \\
\text { from DLS }\end{array}$ \\
\hline S1 & 0.1 & 0.1 & $925 \pm 230$ & - \\
S2 & 0.01 & 0.01 & $103 \pm 35$ & 71 \\
S3 & 0.01 & 0.1 & $175 \pm 45$ & 128 \\
S4 & 0.1 & 0.01 & $37 \pm 13$ & 65 \\
\hline
\end{tabular}

$0.01 \mathrm{M}$ of iron precursor. These solutions were then allowed to react for different periods of time (1-192 h). Particle size and final morphologies were determined using TEM and DLS. The results from these experiments are summarized in Table 1.

\section{Results and discussion}

Independent of initial $\mathrm{FeCl}_{3}$ concentration $\left(\left[\mathrm{FeCl}_{3}\right]\right)$ and subsequent treatment of the solution, all synthesized hematite particles exhibit a cube-like shape (Fig. 1). This is somewhat surprising, as other studies exploring the effect of $\left[\mathrm{FeCl}_{3}\right]$, though under different experimental conditions $(0.02 \mathrm{M})$, indicated that the actual shape of the particles would sensitively depend on $\left[\mathrm{FeCl}_{3}\right][1]$. However, although the shape of the particles remained essentially cube-like in all our experiments, the dimensions of the particles strangely varied with the experimental procedure, as summarized in Table 1 . The resulting particle size distributions all have polydispersities around $20-30 \%$, and the aggregates visible in the TEM images are due to the drying procedure used in the TEM sample preparation process and do not exist in suspension as indicated by the average hydrodynamic radii $R_{h}$ measured by DLS.

The reduction of the initial $\mathrm{FeCl}_{3}$ concentration $\left[\mathrm{FeCl}_{3}\right]_{\text {ini }}$ from $0.1 \mathrm{M}$ (S1, Fig. 1a) to $0.01 \mathrm{M}$ (S2, Fig. 1b) has a dramatic influence on the size of the resulting final nanocubes, which decreases from $d=925 \pm 230 \mathrm{~nm}$ for $\mathrm{S} 1$ to $d=103 \pm 35 \mathrm{~nm}$ for S2. However, the $\mathrm{FeCl}_{3}$ concentration is not the only relevant parameter that determines the final product. This is demonstrated with samples S3 (Fig. 1c) and S4 (Fig. 1d). In the S1- and S2-experiments we maintained the initial volume $(0.1 \mathrm{M}$ and $0.01 \mathrm{M}$, respectively) for the entire reaction time of 7 days during which essentially all starting materials were converted into hematite. In contrast, in the S4-experiment, we diluted the sample after $1 \mathrm{~h}$ to $0.01 \mathrm{M}$; at this point of the reaction the starting material has already been completely converted to akaganeite, but the amount of hematite was still negligible. After this dilution step, the system was then again left to fully convert to hematite for 7 days. Under these conditions the resulting nanocubes in S4 with $d=37 \pm 13 \mathrm{~nm}$ are now much smaller than those obtained when starting directly with an $\mathrm{FeCl}_{3}$ concentration $\left[\mathrm{FeCl}_{3}\right]_{\text {ini }}=0.01 \mathrm{M}$. Similarly, when we started with $\left[\mathrm{FeCl}_{3}\right]_{\text {ini }}=0.01 \mathrm{M}$, and then quenched the reaction and concentrated the sample in a rotavap to an equivalent $\mathrm{FeCl}_{3}$ concentration of $0.1 \mathrm{M}$ before starting the synthesis again, the resulting nanocube size after 7 days, i.e. after full conversion, was now $d=175 \mathrm{~nm}$. This value is about a factor of 1.7 larger than what has been found for S2 (same $\left[\mathrm{FeCl}_{3}\right]_{\text {ini }}$ ), but about a factor of 5.3 smaller than what was obtained with sample $\mathrm{S} 1\left(\left[\mathrm{FeCl}_{3}\right]_{i n i}=\left[\mathrm{FeCl}_{3}\right]_{f}\right)$. This clearly indicates that $\left[\mathrm{FeCl}_{3}\right]$ is not the only size determining factor, but that the size and number density of the akaganeite particles as a precursor phase also play an important role in determining the final dimensions of the hematite particles. In order to explore the role of the akaganeite precursor particles, we thus characterized the shape and size of particles formed $1 \mathrm{~h}$ after the start of the reaction using different initial $\mathrm{FeCl}_{3}$ concentrations. As described above, at this point the bulk of the starting material has been converted to akaganeite for all samples investigated, while no detectable amount of hematite has yet been produced. For the investigated concentration range of $\left[\mathrm{FeCl}_{3}\right]_{\text {ini }}=0.01$ and $0.1 \mathrm{M}$, we found that all akaganeite particles were spindle-shaped, but of different size and aggregation state (Fig. 2a and b).

Depending on $\left[\mathrm{FeCl}_{3}\right]_{\text {ini }}$, akaganeite spindles of various sizes were obtained during the first hour of the synthesis at $98^{\circ} \mathrm{C}$ (Fig. 2a and $b$ ). The average length determined by measuring the long axis of 100 particles (from different meshes on the TEM grid) increases linearly with $\left[\mathrm{FeCl}_{3}\right]_{\text {ini }}$ (Fig. $2 \mathrm{c}$ ). The average aspect ratio appears to be almost independent of the initial precursor concentration (Fig. 2d), with values always remaining between 4 and 5 . In addition to spindles, some star shaped and stacked structures of akaganeite are observed after $1 \mathrm{~h}$ of reaction time (Fig. 2a and b), where the star shaped structures are twinned akaganeite [30]. The relevant precursor particles to consider when trying to understand the final hematite particles shown in Fig. 1 are thus the akaganeite spindles (Fig. 2a and b).

Having established the size and shape of the akaganeite precursor particles that are then converted to hematite in the synthesis routes underlying samples $\mathrm{S} 1-\mathrm{S} 4$, we next performed a

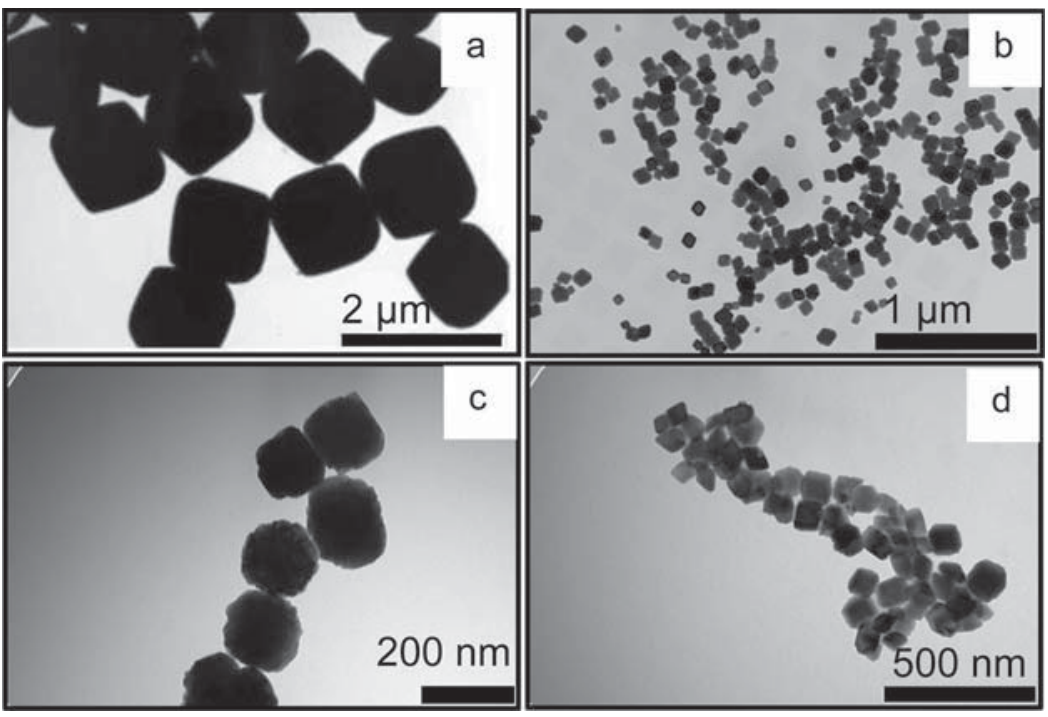

Fig. 1. TEM images of the final hematite cubes obtained in samples (a) S1, (b) S2, (c) S3 and (d) S4. 

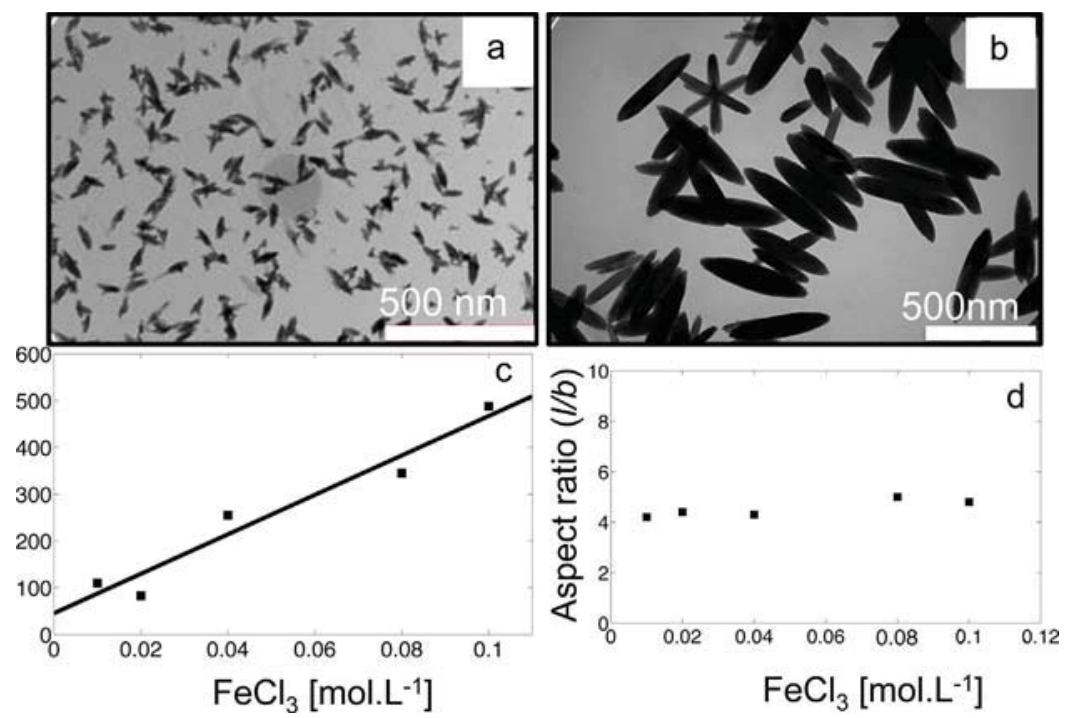

Fig. 2. Properties of the precursor akaganeite spindles as a function of the initial $\mathrm{FeCl}_{3}$ concentration. TEM images of akaganeite spindles synthesized with different $\left[\mathrm{FeCl}_{3}\right]_{\text {ini }}$ values of (a) $0.01 \mathrm{M}$, (b) $0.1 \mathrm{M}$ (scale bar $=500 \mathrm{~nm}$ ). (c) Akaganeite spindle length (long axis) as a function of [FeCl $]_{3}$ ini. (d) Aspect ratio of akaganeite spindles (long axis/small axis) vs. $\left[\mathrm{FeCl}_{3}\right]_{\text {ini }}$.

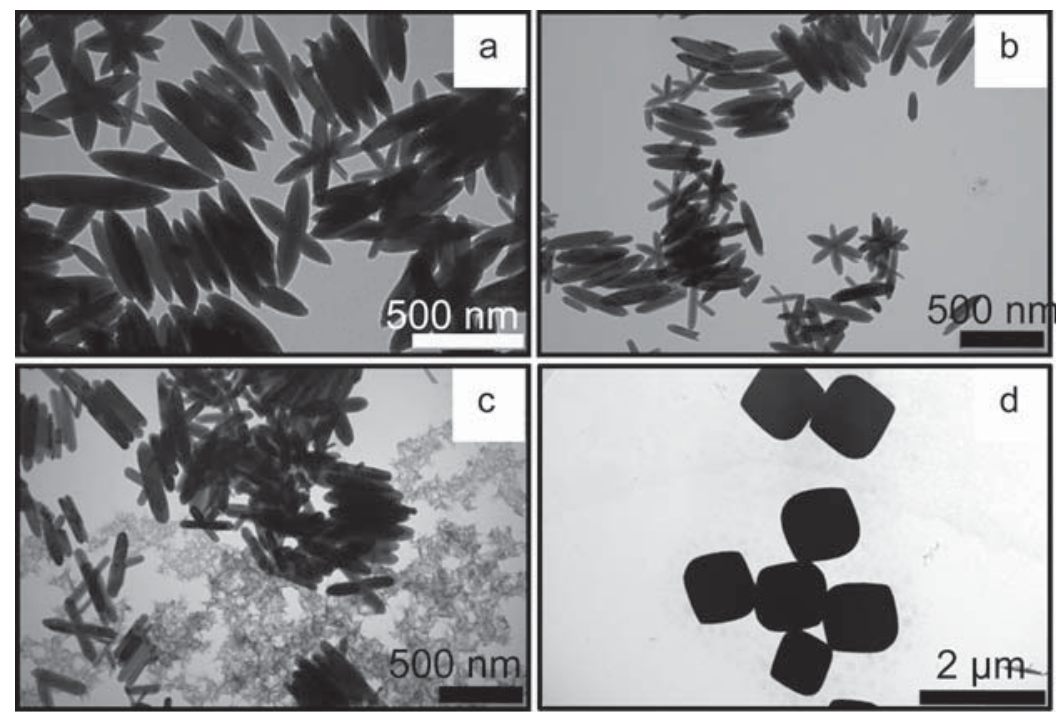

Fig. 3. (a) TEM images of sample $S 1$ after (a) $1 \mathrm{~h}$, (b) $4 \mathrm{~h}$, (c) $24 \mathrm{~h}$ and (d) $192 \mathrm{~h}$.

detailed investigation of the time dependence of the conversion akaganeite-hematite and the morphology of the resulting hematite nanocubes.

In sample $\mathrm{S} 1$ obtained without subsequent dilution $\left[\mathrm{FeCl}_{3}\right]_{\text {ini }}=\left[\mathrm{FeCl}_{3}\right]_{f}=0.1 \mathrm{M}$, large hematite pseudocubical particles are formed (Fig. 3). Our experiments with S1 appear to be in accordance with the earlier findings in Ref. [6]. Our data show that after $1 \mathrm{~h}$ of reaction only akaganeite spindles can be observed which appear to have aggregated into larger stacks or rafts (Fig. 3a). The spindle axes are parallel to the $c$-axis of the $\beta$-FeOOH structure. Diffraction patterns taken with the aperture over an entire raft show that the $c$-axes of the spindles forming a raft are well aligned (Fig. 4a). Moreover, these rafts may contain more than one layer of spindles (Fig. 4b).

The hematite particles nucleate epitaxially on the akaganeite rods (Fig. 5), i.e. the crystallographic orientation of the nuclei is controlled by the akaganeite structure. The following orientation relationship is observed: the $b$-axis of akaganeite is parallel to the

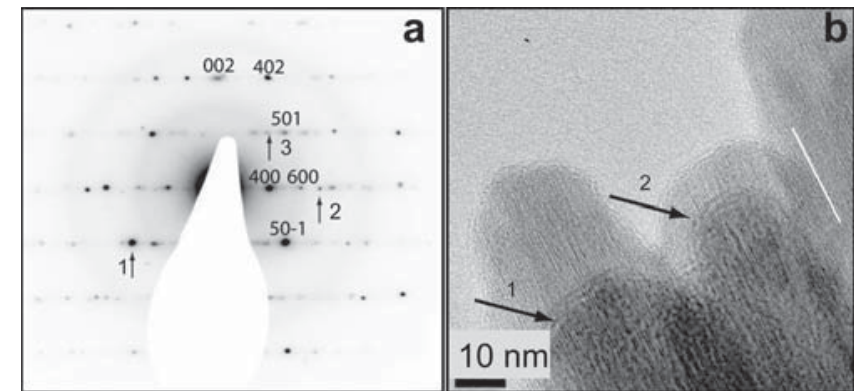

Fig. 4. (a) SEAD pattern from an akaganeite raft. Diffraction spots from two differen rods (indexed spots and arrowed spots) are visible, the $c$-axes of both rods are well aligned, however the $b$-axes are not aligned. (b) TEM image of an akaganeite raft, white line: orientation of the $c$-axis; arrows 1 and 2 point at the two rods, which are either above or below the longer lighter contrasted rods. 


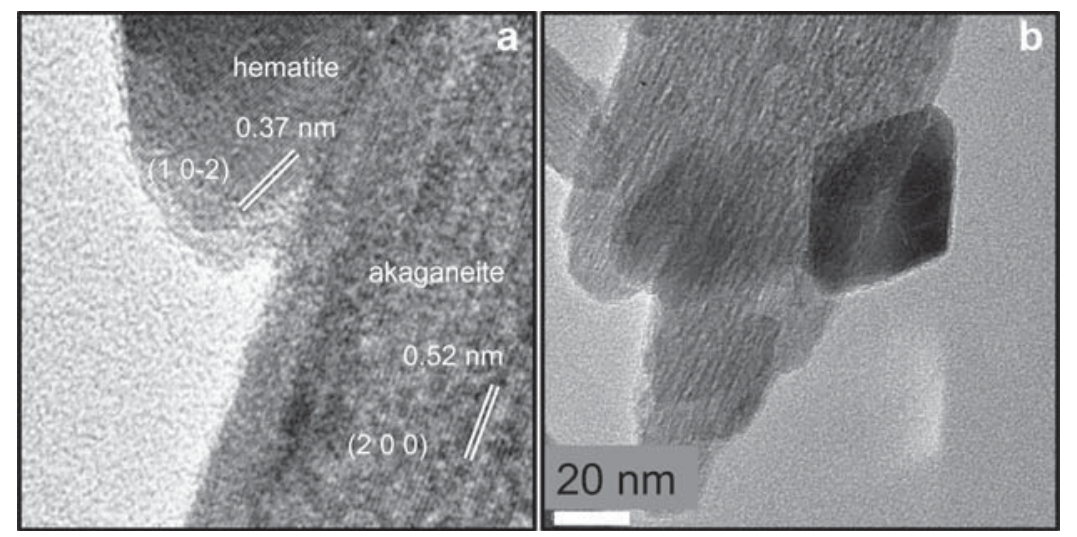

Fig. 5. Detailed view on the initial conversion akaganeite-hematite from HRTEM and TEM: Images are obtained from sample S4 after $1 \mathrm{~h}$ of reaction time. (a) HRTEM image depicting crystallographic orientation of hematite and akaganeite particles. (b) TEM image of a hematite particle, growing on top of a akaganeite raft.

$c$ axis of hematite and the $c$ axis of akaganeite is parallel to the $b$ axis of hematite. The nuclei grow by consuming iron in solution from already dissolved akaganeite. Several hematite nuclei may form within the same stack, and their mutual crystallographic orientation is fixed as long as the akaganeite spindles are held together in a stack.

To some extent there seem to be also topotaxial replacement, as indicated by embayments in the akaganeite surface occupied by hematite. In this process, hematite nucleates in a crystallographically controlled way on the surface of the akaganeite rods and grows into the latter by consuming it. As multiple nuclei form on a single raft, the growing individual hematite nanocubes eventually aggregate/fuse and form a single polycrystalline pseudocube.

Nanocube fusion is supported by Fig. 6 where various crystallographic orientations can be observed in a HRTEM image of a large pseudocube. Furthermore, the solution chemistry enhances the cohesion between the hematite nanocubes. The point zero charge (pzc) increases slightly with particle size from 7.8 for $12 \mathrm{~nm}$ particles to 8.8 for $65 \mathrm{~nm}$ particles as reported by He et al. [31]. The stability ratio as a measure of the colloidal stability against aggregation for nanohematite suspensions drops from 1000 to 1 when increasing the electrolyte concentration from $0.01 \mathrm{M}$ to $0.1 \mathrm{M}$.

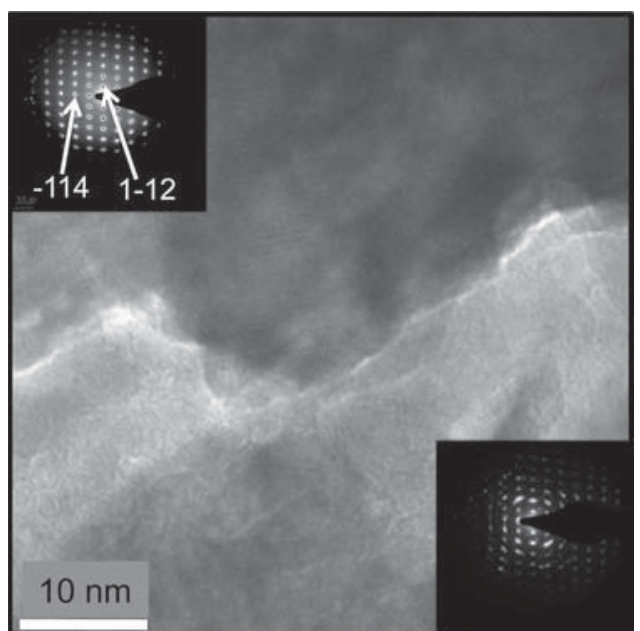

Fig. 6. HRTEM image of sample S1. Shown is a [1 10$]$ lattice fringe image from the boundary between two subcrystals together with two SAED patterns. The upper SAED pattern is taken with the aperture centered over the upper lamella, whereas the lower pattern is taken with the aperture centered over the boundary. The boundary is parallel to the [-114] and [1-12] lattice fringes (arrows show the corresponding diffraction spots).
Nanocubes, which get in contact with each other, will therefore stick together. The growth of the individual cubes is enhanced by the presence of non-aggregated single spindle and small starshaped aggregates, which act as reservoirs that enhance the growth of hematite crystals by Ostwald ripening. This formation scenario for large hematite pseudocubes is in line with the proposed growth model by Bailey et al. [12]. They suggested that for the formation of large pseudocubical particles, akaganeite rods of similar length have first to aggregate into stacks. Nucleation of the hematite then takes place at the interface of the akaganeite rod stacks. The hematite crystals present in the stack coalesce then to a large polycrystalline pseudocubical hematite particles.

The relative orientation of the hematite subcrystals within a pseudocube cannot be observed in a normal TEM experiment due to the large size of the particle. In order to examine the internal nanostructure of the pseudocubic particles, $20 \mathrm{~nm}$ thick slices obtained by sandwiching the pseudocubes in an epoxy resin and cut using a microtome were studied with TEM. Many sections of the hematite particles have a nearly quadratic shape and almost all edges are rounded, but the curvature is not the same for each edge (Fig. 3d). The inner structure of the section is characterized by lath-like subcrystals, delineated by changes in contrast (usually lines of bright contrast) or cracks which radiate out from the center of the section or which are parallel to one external face of the cube, resembling the raft-like arrangement of the primary akaganeite crystal (Fig. 7a). The diffraction patterns taken from different regions of many larger crystals are identical, i.e. the crystallographic orientation does not change across the particle (Fig. 7b-d). Weaker diffraction spots are also present, indicating some misorientation of smaller subgrains, partly an artifact of microtoming.

The observed external shape and internal structure of the hematite cubes are very similar to the features described by Park et al. [32], for monodispersed hematite particles synthesized from highly condensed hydroxide gels [13]. The pseudocubic particles are bound by [012] faces. The internal structure consists of columnar sub-grains that radiate in all directions. Although the orientation of the morphological long axis of the sub-grains changes continuously, the crystallographic orientation from one sub-grain to the next remains the same. The presence of the sub-grains and the constant crystallographic orientation across the particle indicates oriented aggregation of basic particles, in our case primary hematite particles which grew epitactically onto akaganeite rafts (Fig. 5).

Having obtained a detailed description of the formation process and the resulting particle morphology and internal nanostructure of the hematite particles formed at $\left[\mathrm{FeCl}_{3}\right]_{\text {ini }}=0.1 \mathrm{M}$, we next looked at the particle formation at low $\left[\mathrm{FeCl}_{3}\right]_{\text {ini }}=0.01 \mathrm{M}$, i.e. sample S2. 

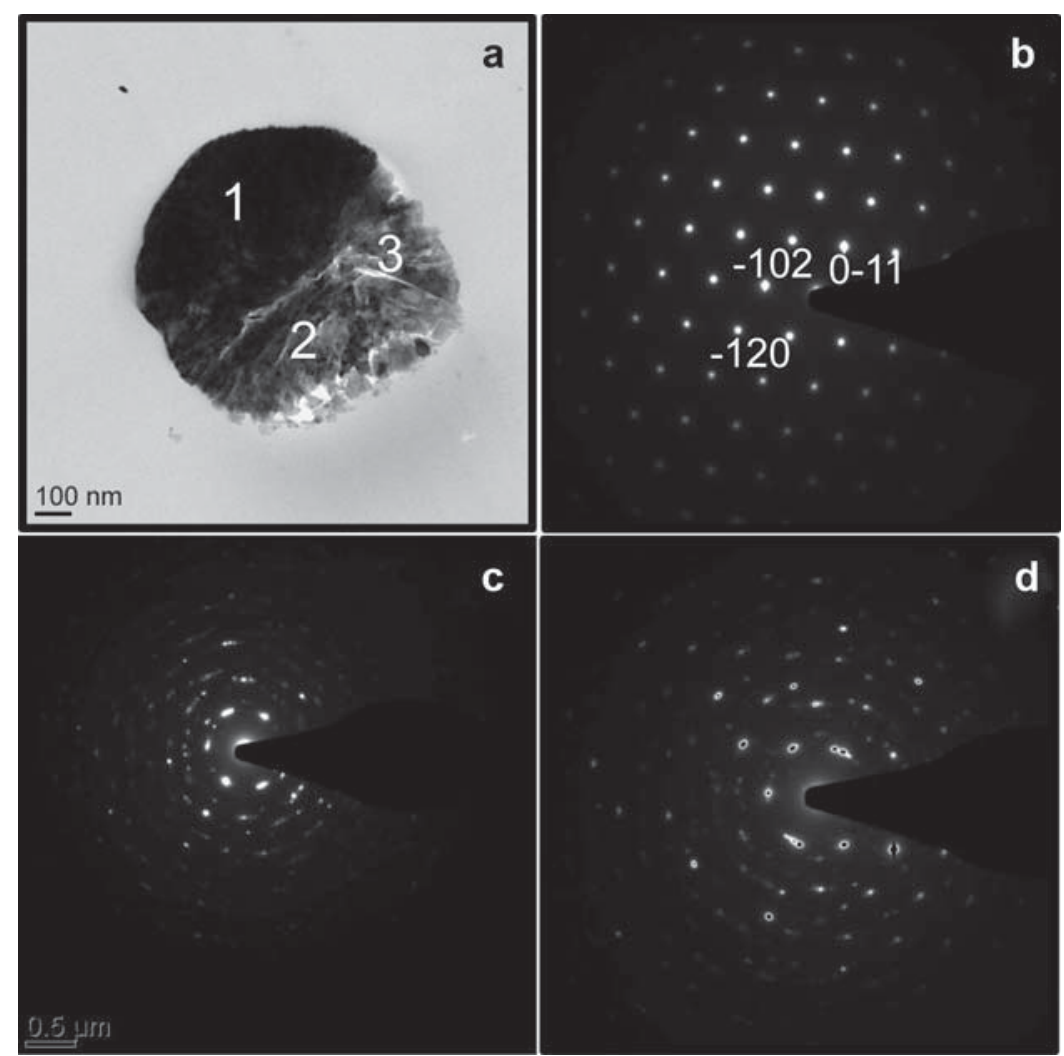

Fig. 7. Details of the interior structure of a large hematite nanocube obtained from sample S1: (a) TEM image of a microtomed large hematite nanocube. (b) SAED pattern from region 1 of hematite nanocube (c) from region 2 and (d) from region 3 of hematite nanocubes. All three diffraction patterns show the same zone axis orientation. The slight streaking is due to rotational disorder caused by microtoming. The long axis of the subcrystal in region 2 is oriented at $90^{\circ}$ with the long axis of the subcrystal in region 3.

As shown in Figs. 2a and 8a, the transformation to hematite occurs via much smaller akaganeite spindles. Akaganeite particles are in fact formed during the first 5 min of the reaction as evident by an XRD powder diffraction analysis (Fig. 2a, supplementary data), and also established by measuring the evolution of the $\mathrm{pH}$ during the synthesis (data not shown). In contrast to the synthesis with $0.10 \mathrm{M}$ initial $\left[\mathrm{FeCl}_{3}\right]$ concentrations, no large rafts are visible after $1 \mathrm{~h}$, and the akaganeite spindles appear to exist mostly as isolated spindles and star-like twinned particles. The lower electrolyte concentration relative to $S 1$ prevents the akaganeite spindles to aggregate. When compared to the temporal evolution of the particles in S1 (Fig. 3), in S2 a significant number of hematite nuclei and smaller nanoparticles are already visible after $4 \mathrm{~h}$ (Fig. 8b). After $24 \mathrm{~h}$ of reaction, cubic and rhomboid-shaped individual hematite crystals are
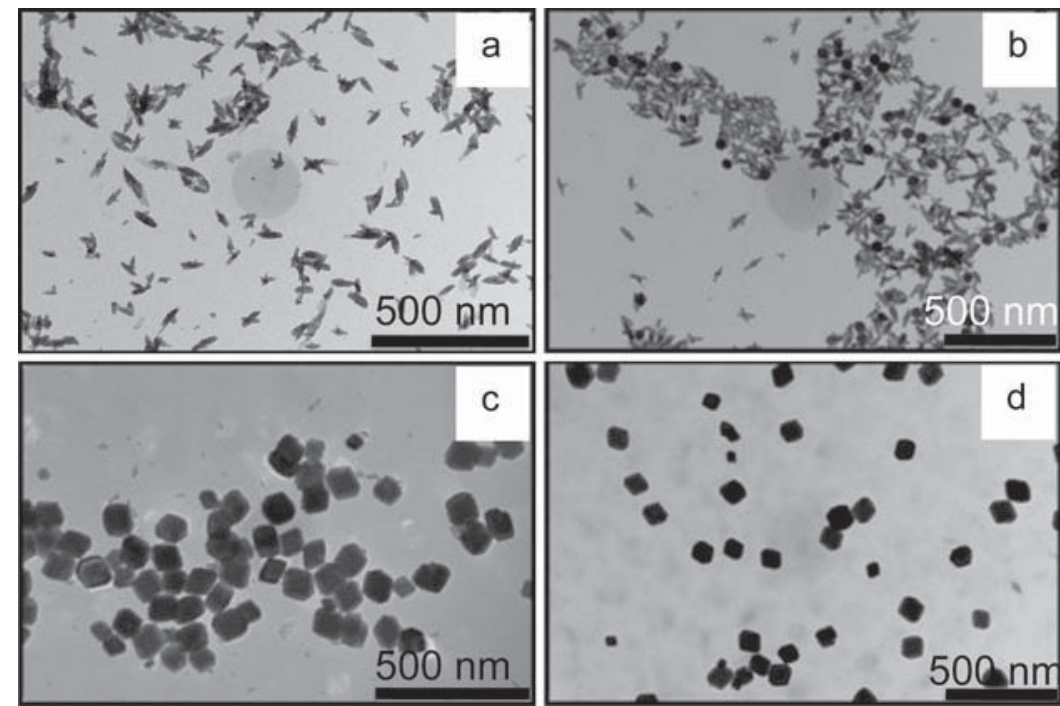

Fig. 8. TEM images of sample S2 after (a) $1 \mathrm{~h}$, (b) $4 \mathrm{~h}$, (c) $24 \mathrm{~h}$ and (d) $192 \mathrm{~h}$. 


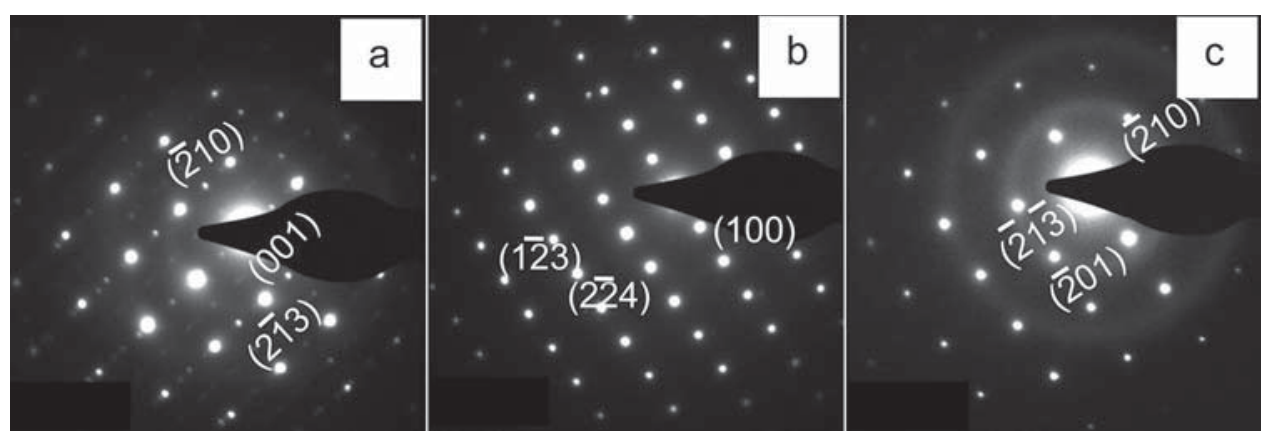

Fig. 9. Selected area electron diffraction (SAED) patterns obtained from hematite nanoparticles through three different routes: (a) S2, (b) S3 and (c) S4.

predominant and only a small amount of remaining small akaganeite debris is left. The akaganeite debris completely disappear after $192 \mathrm{~h}$ of reaction, indicating the full transformation of akaganeite into hematite. The cubic morphology of hematite nanoparticles was also confirmed using SEM (Fig. 1, supplementary data)

The influence of the initial $\mathrm{FeCl}_{3}$ concentration and thus the ionic strength appears to be dramatic. We obtain large polycrystalline hematite pseudocubes in S1 and small nanocubes in S2. Moreover, hematite nanoparticles obtained in S2 single crystals as demonstrated by selected area electron diffraction (SAED) experiments (Fig. 9a). In the spirit of the model proposed by Bailey et al., it is tempting to link the different final size and microtexture to the size of the transient akaganeite crystals and their aggregation state. Akaganeite rods formed at $0.01 \mathrm{M}\left[\mathrm{FeCl}_{3}\right]_{\text {ini }}$ are smaller, but more polydisperse than those formed at $0.1 \mathrm{M}\left[\mathrm{FeCl}_{3}\right]_{\text {ini }}$. The lack of aggregation and stack formation can be explained by the solution chemistry and the surface chemistry of akaganeite. The point of zero charge (pzc) of akaganeite in water is between 7 and 8 , and for $\mathrm{pH}$ values below the pzc the surface of the nanorods is positive. The calculated $\mathrm{pH}$ values for all solutions used in the synthesis of akaganeite have indeed a much lower $\mathrm{pH}$. The stability of the charge stabilized suspensions will thus depend on the electrolyte concentration. At $0.1 \mathrm{M}$ initial $\left[\mathrm{FeCl}_{3}\right]_{\text {ini }}$ concentration and with a high concentration of chlorine anions the surface charge of akaganeite will become effectively screened and aggregation promoted. Subsequent dilution with water will significantly reduce aggregation. The non-aggregated spindles serve as a substrate for the nucleation of hematite, which will grow independently of each other, slowly consuming the smaller akaganeite structures. This mechanism is distinctly different from that proposed at high $\left[\mathrm{FeCl}_{3}\right]_{\text {ini }}$. Apparently the existence of stacks is a pre-requisite for the subsequent formation of larger hematite pseudocubes.

However, it is not only the resulting size and morphology that distinguishes S2 from S1. A comparison of Figs. 3 and 8 also reveals that the transformation kinetics at $0.01 \mathrm{M}\left[\mathrm{FeCl}_{3}\right]_{\text {ini }}$ is much faster, which at first seems counterintuitive. Iron hydroxide phases such as akaganeite and lepidocrocite are metastable phases, which subsequently transform into a stable phase such as hematite or magnetite. The degree of metastability mainly depends on two factors, surface charge and solid-liquid surface free energy. While we are not changing the surface charge when changing $\left[\mathrm{FeCl}_{3}\right]_{\text {ini }}$, the differences in akaganeite particle size result in a significant variation of the surface free energy. If we treat the akaganeite particles as cylinders of finite length $(L)$ and radius $(r)$, the surface free energy per unit volume for S2 akaganeite rods is higher by one order of magnitude than that of $\mathrm{S} 1$, and the large akaganeite particles will thus take much more time to dissolve and transform into hematite.

The results presented so far show that in S2 the formation of hematite is also a two-step process; first $\beta$-FeOOH is formed, which then enables the nucleation and growth of hematite. The resulting hematite particles size and the transformation kinetics for both S1 and S2 are directly linked to the state of the precursor akaganeite particles (size, aggregate state). However, it remains to be seen whether $\left[\mathrm{FeCl}_{3}\right]_{\text {ini }}$ is the only relevant control parameter. We therefore performed two more experimental series using a two-step
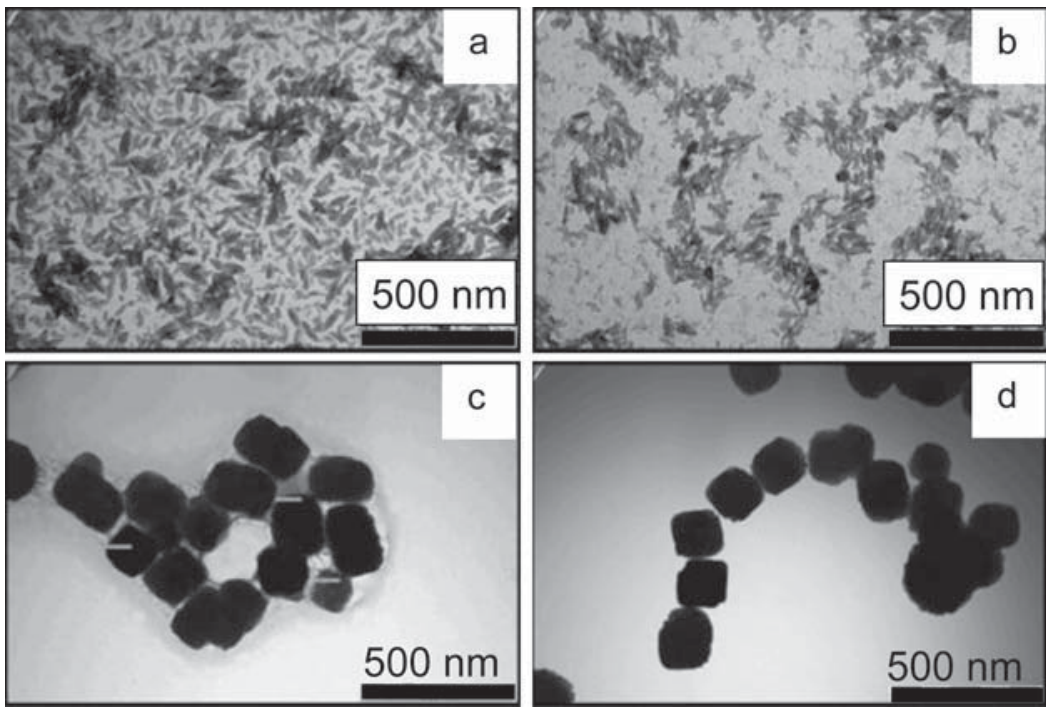

Fig. 10. TEM images of the sample $S 3$ after (a) $0 \mathrm{~h}$, (b) $4 \mathrm{~h}$, (c) $24 \mathrm{~h}$ and (d) $192 \mathrm{~h}$. 

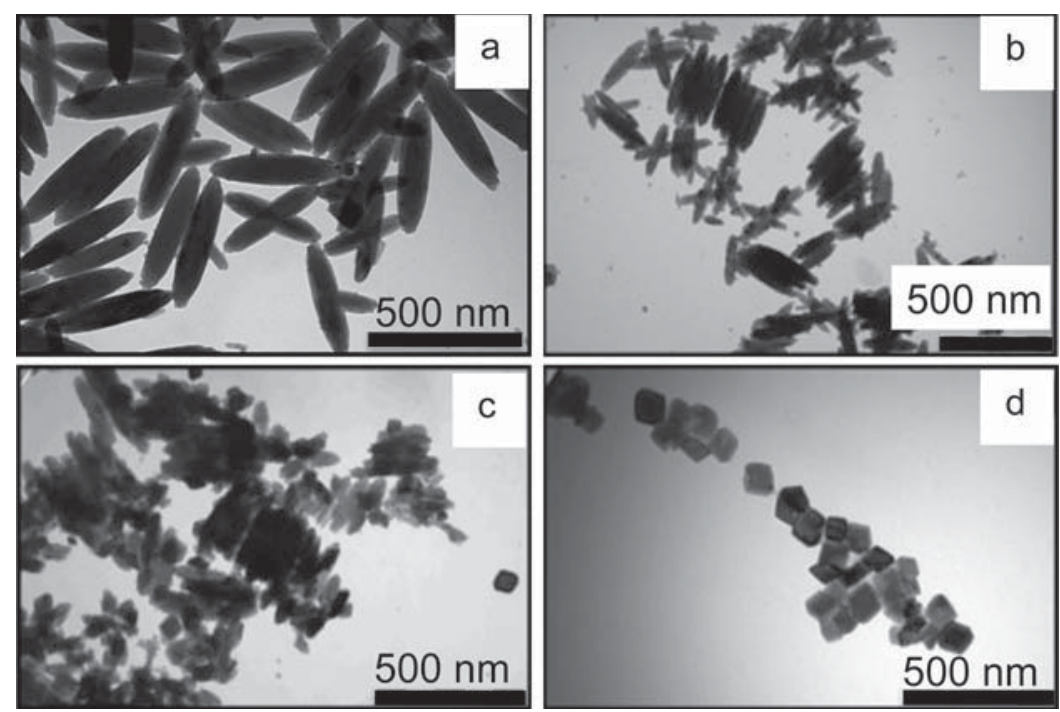

Fig. 11. TEM images of the sample S4 after (a) $0 \mathrm{~h}$, (b) $4 \mathrm{~h}$, (c) $24 \mathrm{~h}$ and (d) $192 \mathrm{~h}$.

synthesis procedure to decouple the role of the precursor particle size and the total amount of precursor material available.

In these two additional series S3 and S4, we study the transformation akaganeite-hematite using a precursor suspension of small akaganeite spindles (prepared at $\left[\mathrm{FeCl}_{3}\right]_{\text {ini }}=0.01 \mathrm{M}$ ) concentrated by a factor of 10 to a high effective or final Fe-concentration (denoted by $[\mathrm{Fe}]_{f}$ ) of $0.1 \mathrm{M}(\mathrm{S} 3)$ and compare it with a transformation of large akaganeite spindles (prepared at $\left[\mathrm{FeCl}_{3}\right]_{\text {ini }}=0.1 \mathrm{M}$ ) diluted by a factor of 10 to a low final Fe-concentration (denoted by $[\mathrm{Fe}]_{f}$ ) of $0.01 \mathrm{M}(\mathrm{S} 4)$. The resulting conversion kinetics and the size and shape of the formed particles are summarized in Figs. 10 and 11.

When we first compare the effect of the number density or $\left[\mathrm{FeCl}_{3}\right]_{f}$ for the small precursor spindles (S2 and S3), we see that despite the 10-fold higher particle concentration the aggregation behavior appears unchanged and the appearance of the sample at early stages is still dominated by individual small akaganeite spindles and a few twins. We observe the first hematite nanoparticles to appear after about $4 \mathrm{~h}$ of reaction (Fig. 10b), quite similar to the situation encountered for sample S2 (Fig. 8b) at a 10-fold lower particle number concentration. The akaganeite spindles appear to have converted completely to hematite nanoparticles after $24 \mathrm{~h}$ of reaction (Fig. 10c), and no change in size and shape of the hematite particles can be observed upon further aging at $98^{\circ} \mathrm{C}$. The particle growth is thus accelerated at the higher concentration when compared to Fig. 8 , where after $24 \mathrm{~h}$ there is still a small but detectable akaganeite population present. Additional TEM experiments (data not shown) show that already after $16 \mathrm{~h}$ of reaction no akaganeite can be detected anymore in S3. There is no evidence that the final hematite particles consist of hematite subunits, and the electron diffraction patterns confirm their single crystalline nature (Fig. 9b). Our experiments thus indicate a growth mechanism in agreement with nucleation and (diffusional) growth rather than an initial formation of stacks of precursor particles that are then converted into hematite nanocubes.

It is interesting to compare the sizes of the final nanocubes. While the procedure followed for S2 leads to hematite nanocubes with an average size of $103 \mathrm{~nm}$, the 10 -fold higher precursor particle concentration in S3 leads to a nanocube size of $175 \mathrm{~nm}$. This corresponds to an increase in particle volume of a factor of 5 . It thus appears as if the total number of particles has only increased by a factor of two, and most of the additional material has been used to grow larger particles, despite the fact that the number concentration of precursor particles has increased by a factor of 10 . A first rather unexpected result of these investigations is thus that the small akaganeite spindles act as nucleation sites for the formation of hematite nanocubes, but that their number is not directly correlated with the number of nuclei. A two-step synthesis procedure as applied in sample $\mathrm{S} 3$ could thus be used as a tool to tune the size of the final hematite nanocubes.

When we next compare the effect of the number density or $\left[\mathrm{FeCl}_{3}\right]_{f}$ for the large precursor spindles (S1 and S4), we still observe the presence of rafts with well aligned stacks of akaganeite spindles at lower total concentration (Fig. 11a). However, multiple hematite nuclei appear to grow now not only on rafts but also on star-like twins. In contrast to the large hematite pseudocubic particles synthesized in sample series S1, Fig. 11d shows that the final particles obtained in S4 are hematite nanocubes with a much smaller size of about $37 \mathrm{~nm}$. Moreover, SAED (Fig. 9c) clearly indicates that the nanocube particles are single crystal in nature. We therefore do not observe a particle synthesis mechanism along the model initially proposed for the hematite particle production at high $(0.10-0.45 \mathrm{M})$ iron salt concentration through a nucleation and growth of multiple nuclei on rafts of akaganeite rods, where large polycrystalline hematite particles form. Instead, nuclei appear on each aggregate or even on individual large akaganeite rods, and lead to the formation of small single crystalline nanocubes. In this case a lower concentration not only suppresses growth as seen for S2 and S3, but at the same time appears to enhance nucleation, leading to the formation of small cubes in S4.

Again it is interesting to compare the initial akaganeite precursor particle size (length a about $500 \mathrm{~nm}$, axial ratio $l / b$ about 5 ) with the size of the final hematite nanocube (cube size about $37 \mathrm{~nm}$ ), which indicates that every precursor particle provides material for a larger number of hematite nanocubes, in this case approximately 60. This two-step synthesis process appears to follow a LaMer model-like mechanism. According to this model, individual nuclei grow by dissolution and precipitation of existing species (akaganeite in this particular case) [33]. Hematite nuclei form in the solution and grow by consuming akaganeite. Our experiments thus demonstrate that the presence of stacks of large akaganeite precursor particles does not necessarily lead to the formation of large polycrystalline pseudocubic hematite particles. It is only in combination with a high mass concentration that we find evidence for this scenario, whereas at lower particle concentrations we still observe the formation of small single crystal nanocubes. 


\section{Conclusion}

We have performed a detailed analysis of the particle growth in $\mathrm{FeCl}_{3}$ solutions under forced hydrolysis conditions at $98^{\circ} \mathrm{C}$, where first akaganeite spindles form that then convert into cubelike hematite particles with time. For the akaganeite spindles we observe that the average length depends linearly on the initial $\mathrm{FeCl}_{3}$ concentration, while the anisotropy is hardly affected by the same parameter. Studying the subsequent conversion into hematite, we find that depending upon the initial $\mathrm{FeCl}_{3}$ concentration either small, single crystal hematite nanocubes or larger, pseudocubical polycrystalline hematite particles form. At first it seems tempting to link the final size, shape and crystallinity of the hematite particles to the different sizes and state of aggregation of the intermediate akaganeite particles. However, our experiments clearly demonstrate that both the particle size and concentration of the akaganeite precursor have a significant effect on the resulting hematite particles, their size and crystallinity.

Although we find rational arguments that allow us for example to interpret the differences in conversion kinetics for small and large precursor particles in terms of their different surface free energy, or the variations seen for different final concentrations as due to a concentration-enhanced growth, we lack a model that provides a consistent description of the findings. However, while the findings made during our study pose new questions with respect to the formation mechanism of cube-like particles that will require more detailed investigations of the nucleation and growth processes using high-resolution techniques, they nevertheless offer new pathways towards better size control for hematite nanoparticles. Following a two-step synthesis procedure, single crystal hematite nanocubes with tunable sizes ranging from $37 \mathrm{~nm}$ to $175 \mathrm{~nm}$ can be obtained by properly choosing the akaganeite precursor dispersion in terms of its particle morphology and concentration. It will certainly be interesting to see whether we can generalize our findings and extend them to other systems, or use the resulting nanocubes as templates for creating more advanced structures for example through an additional coating step with silica.

\section{Acknowledgments}

The authors would like to thank Laurent Mirolo for his help with the XRD measurements. We gratefully acknowledge financial support from the Swiss National Science Foundation and the Adolphe Merkle Foundation. We would also like to thank Kitty van Gruijthuijsen for valuable discussions and suggestions.

\section{Appendix A. Supplementary Data}

Supplementary data associated with this article can be found, in the online version

\section{References}

[1] J.-H. Lee, K. Lee, S.H. Moon, Y. Lee, T.G. Park, J. Cheon, All-in-one target-cellspecific magnetic nanoparticles for simultaneous molecular imaging and sirna delivery, Angewandte Chemie 121 (2009) 4238-4243.

[2] A.-H. Lu, E.L. Salabas, F. Schüth, Magnetic nanoparticles: synthesis, protection, functionalization, and application, Angewandte Chemie International Edition 46 (2007) 1222-1244.

[3] S.V. Sonti, A. Bose, DNA isolation using avidin-coated magnetic nanoclusters, Colloids and Surfaces B: Biointerfaces 8 (1997) 199-204.

[4] V. Malik, A.V. Petukhov, L. He, Y. Yin, M. Schmidt, Colloidal crystallization and structural changes in suspensions of silica/magnetite core-shell nanoparticles, Langmuir 28 (2012) 14777-14783.
[5] H. Le, V. Malik, W. Mingsheng, H. Yongxing, F.E. Anson, Y. Yin, Self-assembly and magnetically induced phase transition of three-dimensional colloidal photonic crystals, Nanoscale 4 (2012) 4438-4442.

[6] V. Malik, K.J. Suthar, D.C. Mancini, J. Ilavsky, Magnetic-field-dependent assembly of silica-coated magnetite nanoclusters probed by ultra-small-angle X-ray scattering (USAXS), Journal of Magnetism and Magnetic Materials 354 (2014) 70-75.

[7] E. Matijevic, P. Scheiner, Ferric hydrous oxide sols. III. Preparation of uniform particles by hydrolysis of Fe (III)-chloride, -nitrate, and -perchlorate solutions, Journal of Colloid and Interface Science 63 (1978) 509-524.

[8] H. Cao, G. Wang, L. Zhang, Y. Liang, S. Zhang, X. Zhang, Shape and magnetic properties of single-crystalline hematite $\left(\alpha-\mathrm{Fe}_{2} \mathrm{O}_{3}\right)$ nanocrystals, ChemPhysChem 7 (2006) 1897-1901

[9] S. Mitra, S. Das, K. Mandal, S. Chaudhuri, Synthesis of a $\alpha-\mathrm{Fe}_{2} \mathrm{O}_{3}$ nanocrystal in its different morphological attributes: growth mechanism, optical and magnetic properties, Nanotechnology 18 (2007) 275608

[10] Z. Pu, M. Cao, J. Yang, K. Huang, C. Hu, Controlled synthesis and growth mechanism of hematite nanorhombohedra, nanorods and nanocubes, Nanotechnology 17 (2006) 799

[11] T. Raming, A. Winnubst, C. van Kats, A. Philipse, The synthesis and magnetic properties of nanosized hematite $\alpha-\mathrm{Fe}_{2} \mathrm{O}_{3}$ particles, Journal of Colloid and Interface Science 249 (2002) 346-350.

[12] J. Bailey, C. Brinker, M. Mecartney, Growth mechanisms of iron oxide particles of differing morphologies from the forced hydrolysis of ferric chloride solutions, Journal of Colloid and Interface Science 157 (1993) 1-13.

[13] T. Sugimoto, K. Sakata, A. Muramatsu, Formation mechanism of monodisperse pseudocubic $\alpha-\mathrm{Fe}_{2} \mathrm{O}_{3}$ particles from condensed ferric hydroxide gel, Journal of Colloid and Interface Science 159 (1993) 372-382.

[14] M. Ozaki, S. Kratohvil, E. Matijevic, Formation of monodispersed spindle-type hematite particles, Journal of Colloid and Interface Science 102 (1984) 146

[15] M. Ocana, M.P. Morales, C.J. Serna, Homogeneous precipitation of uniform $\alpha-$ $\mathrm{Fe}_{2} \mathrm{O}_{3}$ particles from iron salts solutions in the presence of urea, Journal of Colloid and Interface Science 212 (1999) 317

[16] H. Wang, X. Zhang, B. Liu, H. Zhao, Y. Li, Y. Huang, Z. Du, Synthesis and char acterization of single crystal $\alpha-\mathrm{Fe}_{2} \mathrm{O}_{3}$ nanobelts, Chemistry Letters 34 (2005) 184-185.

[17] M. Cao, T. Liu, S. Gao, G. Sun, X. Wu, C. Hu, Z. Wang, Single-crystal dendritic micro-pines of magnetic $\alpha-\mathrm{Fe}_{2} \mathrm{O}_{3}$ : large-scale synthesis, formation mechanism, and properties, Angewandte Chemie International Edition 44 (2005) 4197-4201.

[18] T. Sugimoto, Formation of monodispersed nano- and micro-particles controlled in size, shape, and internal structure, Chemical Engineering \& Technology 26 (2003) 313-321.

[19] T. Sugimoto, Y. Wang, Mechanism of the shape and structure control of monodispersed $\alpha-\mathrm{Fe}_{2} \mathrm{O}_{3}$ particles by sulfate ions, Journal of Colloid and Interface Science 207 (1998) 137-149.

[20] F. Jones, M. Ogden, A. Oliveira, G. Parkinson, W. Richmond, The effect of phosphonate-based growth modifiers on the morphology of hematite nanoparticles formed via acid hydrolysis of ferric chloride solutions, CrystEngComm 5 (2003) 159-163.

[21] P. Riveros, J. Dutrizac, The precipitation of hematite from ferric chloride media, Hydrometallurgy 46 (1997) 85-104.

[22] T. Sugimoto, H. Itoh, T. Mochida, Shape control of monodisperse hematite particles by organic additives in the gel-sol system, Journal of Colloid and Interface Science 205 (1998) 42-52.

[23] A. Mackay, Beta-ferric oxyhydroxide, Mineralogical Magazine 32 (1960) 545.

[24] R. Peterson, H. Rahman, The ion exchange properties of crystalline inorganic oxide-hydroxides. Part I. $\beta \mathrm{FeOOH}$ : a variable capacity anion exchanger, Journal of Colloid and Interface Science 94 (1983) 60.

[25] R. Blender, W. Dieterich, Debye-Huckel theory on random fractals, Journal of Physics A: Mathematical and General 19 (1986) L785.

[26] A. Bapat, C. Perrey, S. Campbell, C. Carter, U. Kortshagen, Synthesis of highly oriented, single-crystal silicon nanoparticles in a low-pressure, inductively coupled plasma, Journal of Applied Physics 94 (2003) 1969.

[27] Y. Lei, W. Chim, J. Weissmüller, G. Wilde, H. Sun, X. Pan, Ordered arrays of highly oriented single-crystal semiconductor nanoparticles on silicon substrates, Nanotechnology 16 (2005) 1892.

[28] N. Ni, M. Tillman, J. Yan, A. Kracher, S. Hannahs, S. Budko, P. Canfield, Effects of co substitution on thermodynamic and transport properties and $\mathrm{H}_{\mathrm{c} 2}$ in $\mathrm{Ba}\left(\mathrm{Fe}_{1-x} \mathrm{Co}_{x}\right)_{2} \mathrm{As}_{2}$ crystals, Physical Review B 78 (2008) 214515.

[29] J.-Q. Yan, A. Kreyssig, S. Nandi, N. Ni, S.L. Bud'ko, A. Kracher, R.J. McQueeney, R.W. McCallum, T.A. Lograsso, A.I. Goldman, P.C. Canfield, Structural transition and anisotropic properties of single-crystalline $\mathrm{SrFe}_{2} \mathrm{As}_{2}$, Physical Review B 78 (2008) 024516

[30] A. Mackay, Debye-Huckel theory on random fractals, Mineralogical Magazine 33 (1962) 270

[31] Y.T. He, J. Wan, Y. Tokunaga, Kinetic stability of hematite nanoparticles: the effect of particle sizes, Journal of Nanoparticles Research 10 (2008) 321-332.

[32] G. Park, D. Shindo, Y. Waseda, T. Sugimoto, Internal structure analysis of monodispersed pseudocubic hematite particles by electron microscopy, Journal of Colloid and Interface Science 177 (1996) 198-207.

[33] V.K. LaMer, R.H. Dinegar, Theory, production and mechanism of formation of monodispersed hydrosols, Journal of the American Chemical Society 72 (1950) 4847-4854. 